\title{
Merits of coincident observation of energetic cosmic events by astronomical and gravity wave observatories
}

Szabolcs Marka

Szabolcs Marka, "Merits of coincident observation of energetic cosmic events by astronomical and gravity wave observatories," Proc. SPIE 4856, Gravitational-Wave Detection, (26 February 2003); doi: 10.1117/12.459091

Event: Astronomical Telescopes and Instrumentation, 2002, Waikoloa, Hawai'i, United States 


\title{
Merits of coincident observation of energetic cosmic events by astronomical and gravity wave observatories
}

\author{
Szabolcs Márka* \\ LIGO $^{\dagger}$ Laboratory ${ }^{\ddagger}$, California Institute of Technology, Pasadena, California, USA
}

\begin{abstract}
Presently there are six interferometric gravitational wave detectors in the commissioning or construction phase in North America, Europe, and Japan. Once completed this worldwide network of detectors will be capable of detecting gravitational waves with unprecedented detail and sensitivity. Their ambition reaches well beyond the first direct detection of gravitational waves; they promise the dawn of a new field, the gravitational wave astronomy. One of the major goals of interferometric gravity wave detectors is to develop and exploit gravitational wave detection in conjunction with other conventional observational techniques, which are capable of observing the same astronomical process using different methods. The most promising areas are the optical, GRB and neutrino searches for energetic processes. Coincident observation of astronomical events shall revolutionize the way we understand energetic processes and will provide a new window on compact and difficult to study astronomical objects such as stellar cores. We will discuss the status, the potential future, and benefits of collaboration amongst gravitational wave detector networks and astronomical/GRB/neutrino networks and some of the practical experiences with the LIGO detectors.
\end{abstract}

Keywords: supernovae, neutrino, GRB, gravity wave, gravitational radiation, SNEWS, GCN, IPN

\section{INTRODUCTION AND SCIENTIFIC MOTIVATION}

It is a often believed that the goal of interferometric gravitational wave detectors ${ }^{[1]}$ is the first direct detection of gravitational waves, while in reality the first detection is only one of the milestones. Interferometric gravitational wave detectors will likely get the chance to experience the excitement of the first direct detection. However, that will only be the beginning of a new exciting field of gravitational wave astronomy ${ }^{[2]}$. These detectors are designed and built ${ }^{[3]}$ to be suitable for observation of large gravity wave events. Efforts are also being made to ensure that these detectors operate in close collaboration with each other to ensure the best possible scientific results. An overview of the status and the possible scientific reach of interferometric gravitational wave detectors is given in Ref. [2].

Eventually these detectors will become one of the common astronomical tools, providing a fundamentally different view of the universe. This new data is complementary to the information obtained via more traditional observational tools. The differences between electromagnetic, neutrino and gravitational wave properties, propagation and emission are very pronounced. Even though each can accompany the same astronomical event, they are usually emitted at different times by different sub-processes. Therefore each channel carries "proprietary" information about the pieces of a big cosmological "puzzle".

In contrast to electromagnetic radiation, gravitational waves do not interact strongly with matter, which makes the universe transparent for gravitational waves. This transparency allows direct observation of processes that are out of the reach of the traditional instruments (e.g. supernovae hidden by the galactic center). In practice, one can only achieve

\footnotetext{
* smarka@ligo.caltech.edu; phone 1626395 2005; www.ligo.caltech.edu/ smarka; Address: Szabolcs Márka, LIGO Laboratory, California Institute of Technology, MS 18-34, Pasadena, CA 91125, USA

${ }^{\dagger}$ Detailed information about the Laser Interferometer Gravitational Wave Observatory (LIGO) is available at http://www.ligo.caltech.edu.

${ }^{*}$ Detailed information about the LIGO Scientific Collaboration (LSC) is available at http://www.ligo.org.
} 
partial coverage of the sky at any given moment using the electromagnetic channels. On the other hand, each interferometric gravity wave detector can cover nearly the full solid angle at any given time. Of course this blessing comes at a price. We need a global network of gravity wave detectors with perfect timing to obtain polarization and directional information. Alternatively, coincident observation of an event in the gravity wave and electromagnetic or neutrino channels can also provide us with directional information.

It will become quite natural to consider gravitational wave events as an important piece along with the diverse set of coincident observations in multiple channels. There is a rich history of rewarding cooperation of gravity wave searches and related fields. It is enough to mention Hulse and Taylor's success ${ }^{[4]}$ in measuring the orbital period of PSR $1913+16$ and comparing it to predictions from general relativity. More recent results ${ }^{[5]}$ are also available, which place bounds on the graviton's mass based on binary pulsar observations. Coincidently, one of the major goals of the Laser Interferometer Gravitational Wave Observatory (LIGO) and other interferometric gravitational wave observatories is to develop and exploit gravitational wave detection in conjunction with various independent observations ${ }^{[6]}$, which will prove to be profitable for all parties involved.

A network of optical, neutrino and gravity wave detectors will be able to alert each other when an energetic event with possible cosmic origin is detected. For example, neutrino experiments already have reliable triggers for galactic events and they can distinguish a neutrino burst from background processes. In contrast, it might be difficult to trigger directly on a weak gravity wave event, since the gravity wave signature of possible burst sources is not well understood yet. Thus neutrino experiments are in a position to provide reliable triggers, cross checks and verification for first generation gravity wave searches. In return interferometric gravitational wave observatories will allow novel measurements, which are important for astronomers and neutrino scientists. In the long term, advanced gravity wave detectors will have extragalactic reach, and thus they will be able to provide triggers for astronomers in case of more distant sources besides supplying vital and otherwise unobtainable information regarding the source dynamics.

Exciting science can be learned from sources within our galaxy, which lie in the overlapping range of neutrino, electromagnetic and gravity wave detectors. This is the range where correlated measurements can be most profitable. For example it will help to draft a more solid picture of the dynamics of a core collapse as various phases of the core dynamics will be inscribed in the gravitational wave signature of the event; an absolutely fascinating and detailed record of the details of the core the collapse. Clearly, these measurements heavily rely heavily on the less likely but much more spectacular nearby collapses. It is likely that the observation of a galactic event in multiple observation channels will completely revolutionize the way we understand its dynamics and origin, therefore it is worth waiting (even decades) for this opportunity to gain such exciting knowledge.

\section{ON THE MERITS OF COINCIDENT OBSERVATIONS}

\section{Supernova Searches}

All likely sources of gravitational waves offer a good opportunity for collaboration between astronomical and gravity wave detectors. Binary inspiral searches will finally give a tool to astronomers to study a large but quite elusive population of exotic objects. With the sensitivity level of advanced interferometric gravitational wave detectors, scientist can have the chance to study and classify the compact binary population of the nearby ${ }^{[7,8]}$ universe. Coincident observation of continuous wave sources, like non-axisymmetric pulsars, will help to deepen our understanding of their dynamical behavior and it can lead to precision measurements of their dynamical properties. Joint efforts in searches for supernova events, as one of the interesting and demonstrative examples, will be discussed in a greater detail in this section.

The core collapse of massive stars should involve a rich dynamics of heavy matter. Therefore we can expect that supernovae can be strong emitters of gravitational waves. However, we are just not sure how strong this radiation is, since the details of core collapses are not very well understood. The strength and therefore the range of gravitational wave (GW) radiation from a "boiling" neutron star is expected to be comparable to those from core collapses. Stronger signals can be produced by "hang-up" processes ${ }^{[9]}$ when the core is transformed into a rapidly rotating bar-like object. Unfortunately it is not clear what fraction of supernovae would display such a behavior. As an example, it is believed that the LIGO range for a "hang-up at $100 \mathrm{~km}$ " can be as large as $\sim 15 \mathrm{Mpc}$, while Advanced LIGO might be able to detect such events from hundreds of Mpc. 


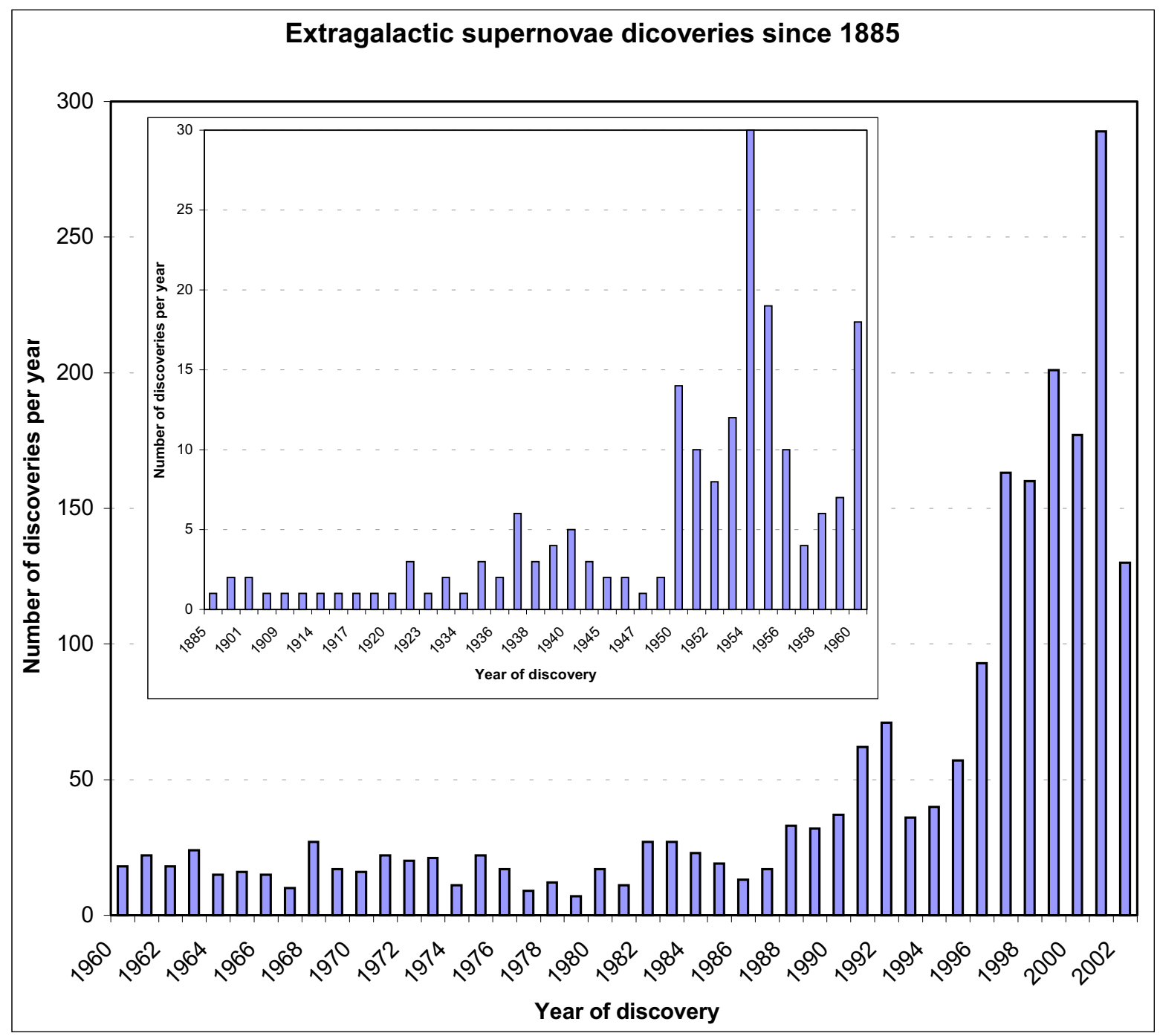

Figure 1 The number of extragalactic supernovae discovered per year since 1885 as of July 2002. Note that the number of discoveries dramatically increased in the last decade, getting close to the 1 /day rate. This graph covers all types of supernovae and only a subset of events has known host galaxy.

Courtesy of Central Bureau for Astronomical Telegrams (http://cfa-www.harvard.edu/cfa/ps/lists/Supernovae.html) and the International Supernovae Network (http://www.supernovae.net/sneyear.htm).

The detection of core collapse is a variation of the chicken and the egg problem. One of the strong motivations for gravity wave searches is that it can facilitate the understanding the dynamics of core collapses. However, we cannot really know what to expect in the gravity wave channel until we understand dynamical details of the compact objects emitting these waves. Certainly, a coincident observation of a "close by" supernova would greatly enhance our confidence and understanding. To overcome this initial difficulty, there is a formidable simulation effort targeting core collapses. Detailed information about the numerical models and relevant results are available from Refs. [9 - 25]. Some of these numerical models are investigated in such detail that the authors ${ }^{[17,18,20]}$ can actually predict large numbers of expected gravitational wave templates and classes. 
In spite all of the advances, there are fundamental questions, which are far from being answered. The expected rate of detectable events is quite uncertain. We know that certain kinds of core collapses can produce strong gravitational waves, but we don't know how common such events are. We expect that type II supernovae emit gravity waves, but the strength of the emission is an open question. Quite a bit of uncertainty is associated with the expected overall rate of supernovae, especially the nearby galactic variety.

Figure 1 shows the discovery rate of extragalactic supernovae for the last century. It is promising that the discovery rate greatly increased in the last decade, nearly reaching the rate of a discovery per day. Unfortunately, it is very likely that most of these extragalactic events are out of reach of the first generation of gravity wave detectors.

The characteristic amplitude of the gravitational wave signal emitted by a type II supernova core collapse strongly depends ${ }^{[19]}$ on the uncertain value of anisotropy of the collapse. Estimates for this anisotropy can be derived using models based on observed pulsar recoil velocities. These very optimistic estimates ${ }^{[16]}$ can predict ranges as large as $\sim 3 \mathrm{Mpc}$ (M81) for LIGO and $15 \mathrm{Mpc}$ (Virgo cluster) for Advanced LIGO. Other, probably more realistic, range estimates ${ }^{[21-24]}$ were derived, based on the Zweiger-Müller catalog of simulated supernova signatures in the gravitational wave band. According to these references, it is very unlikely that first generation gravitational wave detectors will see core collapses from much farther than the edge of our galaxy. The practical range of supernova search will likely fall between the above estimates. Estimated rate of galactic supernova events is one every 30 to 50 years (.033-.02 per year $)^{[19]}$, based upon rates of pulsar formation.

There is another fundamental difficulty in coincident observation of optical supernovae and their gravity wave counterpart. Unlike the neutrino pulse, which is emitted shortly after the gravitational waves, the optical pulse might be delayed as much as several hours relatively to the gravity waves arrival time. In addition, this delay might change from event to event. It makes the coincident detection of optical and gravity waves arising from core collapses very difficult for weak signals. Statistical methods, relying on well-known arrival times are very hard to apply when we have to consider such an uncertainty in the trigger time. Nonetheless, since the gravity wave signal with galactic origin is supposed to arrive in conjunction with a neutrino burst such coincident observation can be linked later to an optical counterpart.

Gravitational waves and neutrinos ${ }^{[26-31]}$ provide new windows on compact and difficult to study astronomical objects like stellar cores. Both types of radiation are emitted promptly during a supernova event and can travel to a distant detector with little attenuation, interaction, or deviation from a straight-line path.

Large bursts of neutrinos and GW are emitted as the core of a massive star collapses in a Type II and possibly Type Ib supernovae. Current observations and modeling results indicate that an asymmetric core collapse is likely, which significantly increases the probability of strong GW emission. According to current theory, the gravitational waves probably precede the neutrino burst in a Type II supernova by an insignificant interval of time. They are released respectively at $.1 \mathrm{~s}$ and $.4 \mathrm{~s}$ after the core collapse begins. Gravitational radiation is probably emitted in a short burst of order of $50 \mathrm{~ms}$.

Each event is a unique one-time episode. Comprehensive and complementary measurements are needed to provide the complete scientific picture. Since the neutrino and gravitational wave pulse is emitted nearly simultaneously, neutrino observatories can also provide high quality triggers for gravity wave searches.

Leading neutrino observatories have somewhat more than a galactic reach and it is very unlikely that it will substantially increase in the foreseeable future. The high price of instrumentation limits the size of neutrino experiments. The Next Neutrino and Nucleon Decay Detector (NNN) (presently in the early planning stage) might have a total mass of one $\sim$ Megaton and its maximum reach for supernovae can be around $1.3 \mathrm{Mpc}$, essentially limiting highly reliable observations to the Local Group. When reaching their design sensitivity, the first generation of gravity wave detectors will have a galactic reach for non-axisymmetric core collapse, which is quite compatible with the range of large neutrino detectors. Some neutrino detectors can also provide directional information. A realistic calculation (including noise) has been made on the pointing accuracy obtained through neutrino-electron elastic scattering ${ }^{3}$ used in the Super-K and SNO experiments. For a supernova at $10 \mathrm{kpc}$ a total of 4400 neutrino interactions occur, of which $\sim 200$ elastic scattering events can be used to obtain directional information. This yields a pointing resolution of 5 degrees for Super-K at 10 $\mathrm{kpc}^{[29]}$. For SNO at $10 \mathrm{kpc}$ the resolution is estimated to be 20 degrees ${ }^{[29]}$.

Coincident detection of neutrino and gravitational wave signals originating from the same source could allow us to measure fundamental properties of supernovae, gravitational waves and neutrinos. A good example of a possible direct measurement of the neutrino mass is described in detail in Ref. [26, 30]. 
A good working example of coordination between different detectors is Supernova Early Warning System (SNEWS) ${ }^{[27}$,

${ }^{28]}$. It is the group of large neutrino experiments with the goal of providing a prompt alert of a supernova occurrence based on a multiple coincidence. The natural beneficiaries are the neutrino observatories themselves, the electromagnetic observatories, gravitational wave observatories and amateur astronomers. SNEWS utilizes the prompt neutrino radiation from a supernova, to give advance real time warning to the scientific community. SNEWS can also serve as a platform for downtime coordination, timing verification and other cooperative activities. The basic coincidence strategy of SNEWS is very effective since individual detector false alarms are due almost entirely to instrumental problems, which are nearly always uncorrelated among different experiments. SNEWS requires that the single detector false alarm rate is equal to or less than one per week for every active participant. After requiring the coincidence of only two detectors with uncorrelated false-alarm rates the net false alarm rate becomes 1 per century when using a 10s coincidence window.

LIGO is able to receive alerts from SNEWS at the present and the full dataset is stored securely, which allows us to start a prompt analysis upon receiving a SNEWS alarm. We do not have an automatic burst search engine in place yet, which can satisfy the stringent false alarm rate requirements of SNEWS. However, it is quite conceivable that one will be developed in the near future.

\section{Gamma-Ray Burst (GRB) ${ }^{[32-46]}$ Searches}

Gamma-Ray Bursts (GRB) ${ }^{[32-34]}$ are short but very energetic pulses of gamma-rays emitted at cosmological distances. They seem to originate from random sky locations. They are quite frequent and their detection rate can be as high as one event a day. According to our present knowledge they are the result of various ultra-relativistic processes, and can be accompanied by X-ray, radio and/or optical afterglows. GRBs require very energetic sources $\left(10^{51}-10^{53} \mathrm{erg}\right)$, and can be as short as $10 \mathrm{~ms}$ and as long as $100 \mathrm{~s}$.

GRBs can be classified based on their duration as "short" $(<2 s)$ and "long" $(>2 s)$. This classification reflects the difference in models used to explain the behavior these two groups. Present estimates of the "long GRB" rate is $\sim 2$ Gpc ${ }^{3} \mathrm{yr}^{-1}$. The rate for short burst is more uncertain since we do not have any observed afterglow associated with a "short GRB", therefore, we lack a reliable distance scale. Current estimates place the rate in the order of $\sim 20 \mathrm{Gpc}^{-3} \mathrm{yr}^{-1}$.

The present consensus is that GRB emission is associated with black hole formation processes such as compact binary inspirals and Collapsars ("failed supernovae"). These sources are exactly the types that are traditionally associated with gravitational waves. Therefore we have a very good reason to expect strong association between GRBs and gravitational waves. There is a large number of good publications available describing possible associations between GRBs and gravity waves (Refs. 32-46).

Considering that compact binary mergers are associated with "short GRBs" and taking into account the currently assumed distance scale of the "short GRBs", one can expect one observable event per year rate for Advanced LIGO (assuming that the present models are accurate). The detection and association of gravitational waves with "long GRBs" is less likely in the near future ${ }^{[32,35]}$.

LIGO pursues an active program concerning coincidence studies between GRBs and gravity waves. LIGO has connections to two major networks specializing in distribution of GRB observational data. We receive timely notices regarding recent GRB events through the Internet. This also allows for prompt and automatic reaction on our part besides the off-line analysis.

- The GRB Coordinates Network $(\mathrm{GCN})^{\S}$ is an Internet based near real time distributor of GRB reports, relying on various observations and other GRB networks. It also serves as a distributor of follow up observation reports supplied by the GRB community. The GCN near real time reports are very detailed and they contain a comprehensive set of known and trusted parameters of the observed events, including accurate directional, strength and timing information.

- The InterPlanetary Network (IPN) ${ }^{* *}$ relies on a (dynamically changing) formation of spacecrafts equipped with GRB detectors. IPN derives the position information via triangulation, utilizing the varying arrival time of the burst at each spacecraft. Presently it receives data from the Ulysses ${ }^{\dagger \dagger}$, Konus-Wind ${ }^{\sharp}$, Mars Odyssey ${ }^{\S \S}$ and

\footnotetext{
${ }^{\S}$ Detailed description of GCN is available at their homepage at: http://gcn.gsfc.nasa.gov/gcn/

${ }^{* *}$ Detailed description of IPN is available at their homepage at: http://ssl.berkeley.edu/ipn3/

${ }^{\dagger \dagger}$ Information on the Ulysses project is available at http://heasarc.gsfc.nasa.gov/docs/heasarc/missions/ulysses.html

\# Information on the Konus-Wind project is available at http://heasarc.gsfc.nasa.gov/docs/heasarc/missions/wind.html
} 
HETE- ${ }^{* * *}$ : FREGATE detectors. Timely reports, available via the Internet, contain a comprehensive set of parameters of the observed events. The nature and precision of directional information depends on the source location and the number of spacecrafts observing the event.

Matching individual GRB events to gravitational wave events is worth pursuing, however, there is an alternative, more sensitive approach. Since GRB events are detected with a relatively high rate, and there is a significant number of recorded events overlapping with times when gravitational wave observatories were collecting data.

As described in Ref. [43], it is possible to cross-correlate the data streams recorded at distant gravitational wave observatories, while taking into account the direction and arrival time reported by the GRB networks. For each GRB trigger, the gravitational wave data streams can be cross-correlated for the time of the predicted by GRB trigger (ONtrigger) and for intervals excluded by the GRB trigger (OFF-trigger). After observing a large number of events, one can build up the ON and OFF-trigger distributions. These distributions then can be compared using standard statistical tools to detect association between GRBs and gravity waves. Modified versions of this method were used in the past for examining associations between GRB events and gravitational wave data recorded by various resonant bar detectors, described in Ref. [44-46]. At LIGO we have an on-going program utilizing the above method using high quality gravitational wave data collected during the engineering/science runs and GRB triggers supplied by the GCN and IPN collaborations.

\section{CONCLUSION}

The international network of gravitational wave detectors will be capable of detecting gravitational waves with unprecedented detail and sensitivity. Coincident observation of astronomical events by gravitational wave detectors and traditional observatories will greatly improve our understanding of energetic cosmic processes and will open a new window on compact and difficult to study astronomical objects such as stellar cores. Interferometric gravitational wave detectors were designed and built to be able to record detailed signatures of large gravity wave events with a realistic rate.

There is a history of collaboration between gravitational wave searches and projects utilizing more traditional tools and observation channels. In the present there are ongoing efforts within the collaborations building interferometric gravitational observatories to utilize information provided by optical, X-ray and radio astronomers, cosmic neutrino searches and GRB observatories. Cooperation in each field presents its own technical and scientific advantages and challenges.

There is a relatively high rate of optical supernovae and GRB discoveries. Uncertainties in our understanding of the details of the underlying processes make the analysis for the optical case model dependent especially when considering the present sensitivity of our gravitational wave detectors. Statistical analysis using GRB triggers is an ongoing effort and the theoretical/modeling support is rapidly increasing. The use of triggers from cosmic neutrino observatories are more straightforward to exploit but the expected detection rate is extremely low since neutrino observatories are only sensitive to galactic events. A galactic event, however, is likely to result in very detailed observations with rich structure, where the scientific payoff can be spectacular.

Up to date the gravitational wave detectors are mostly on the receiving end of the information pipeline but it is clear that in the not too far future they will provide valuable data for the observation community.

\section{ACKNOWLEDGMENTS}

The LIGO Project and LIGO Laboratory were constructed by the National Science Foundation under cooperative agreement PHY-9210038. The Laboratory operates under cooperative agreement PHY-0107417. We are grateful to external organizations and to scientists helping us with data related to our field of study. We are grateful to Scott Barthelmy and the GCN network and Kevin Hurley and the IPN network for providing us with near real time GRB triggers and to the Ulysses, Konus, SAX, and HETE experiments who detect and generate the events distributed GCN and IPN. We thank Kate Scholberg and the Supernova Early Warning System (SNEWS) for providing us with near real time access to relevant neutrino burst information. We would like to thank the people on the LIGO and LSC team, who

$\S \S$ Information on the Mars Odyssey project is available at http://mars.jpl.nasa.gov/odyssey/

*** Information on the HETE-2 project is available at http://space.mit.edu/HETE/ 
are building the instruments, which will allow us to pursue such exciting line of analysis. The author greatly acknowledges the collaborative effort help and suggestions of the people condensing in and around the Burst Upper Limit Group within LSC. I would like to thank Zsuzsa Márka, Benoit Mours, Gary Sanders and Hareem Tariq for their useful comments.

\section{REFERENCES}

1. P. R. Saulson, "Fundamentals of Interferometric Gravitational Wave Detectors", 40-42 and 270-273, (1986), World Scientific, River Edge, NJ.

2. S. Hughes, et. al., "New physics and astronomy with the new gravitational-wave observatories", in Davidson and Quigg eds. The future of Particle Physics, 2001 Snowmass Meeting; Related online version: http://lanl.arXiv.org/abs/astro-ph/0110349

3. B. Barish, "The Detection of Gravitational Waves with LIGO", http://lanl.arXiv.org/abs/gr-qc/9905026

4. R. Hulse and J. Taylor, Astrophys. J. 195, L51 (1975)

5. S. Finn and P. Sutton, "Bounding the mass of the graviton using binary pulsar observations", Phys. Rev. D, V65, 044022, (2002)

6. B. Allen et. al., LIGO Data Analysis White Paper Draft IV, October 1999.

7. A. Lazzarini and W. Majid, "Galactic Distribution in Nearby Sky", LIGO-T980090-00-E, http://www.ligo.caltech.edu/docs/T/T980090-00.pdf

8. B. Tully, "Nearby galaxies catalog", Cambridge and New York, Cambridge University Press, 1988, 221 p, (1988)

9. K. New, "Gravitational Waves from Gravitational Collapse”, http://xxx.lanl.gov/abs/gr-qc/0206041

10. V. Ferrari, "Gravitational Wave Background from a Cosmological Population of Core-Collapse Supernovae", http://xxx.lanl.gov/abs/astro-ph/9804259, Mon.Not.Roy.Astron.Soc. 303 (1999)

11. Y. Gürsel and M. Tinto, "Near optimal solution to the inverse problem for gravitational-wave bursts", Physical Review D 40, 3884-3938 (1989)

12. J. Novak and J. Ibanez, "Gravitational Waves from the Collapse and Bounce of a stellar core in tensor-scalar gravity", http://xxx.lanl.gov/abs/astro-ph/9911298, Astrophys.J. 533 (2000)

13. A. Burrows and J. Hayes, "The Physics of Core-Collapse Supernova Explosions", Proceedings of the 17'th Texas Symposium on Relativistic Astrophysics, http://xxx.lanl.gov/abs/astro-ph/9506060

14. A. Burrows and J. Hayes, "On the Nature of Core collapse Supernova Explosions", Astrophys.J. 450 (1995) 830

15. A. Burrows, "New insights into Core-Collapse Supernova Theory", http://xxx.lanl.gov/abs/astro-ph/9706137

16. S. Nazin and K. Postnov, "High Neutron Star Birth Velocities and Gravitational Radiation during Supernova Explosions", Astron.Astrophys. 317 (1997) L79-L81

17. T. Zweger and E. Müller, "Dynamics and gravitational wave signature of axisymmetric rotational core collapse", Astronomy and Astrophysics 23.9. (1996)

18. H. Dimmelmeier et al., "Relativistic simulations of rotational core collapse. I. Methods, initial models, and code tests", http://xxx.lanl.gov/abs/astro-ph/0204288, "Relativistic simulations of rotational core collapse. II. Collapse dynamics and gravitational Radiation", http://xxx.lanl.gov/abs/astro-ph/0204289

19. B. Allen et al., Subgroup Report: Astrophysical Source Identification and Signature, LIGO-G000061-00-D, March 1999.

20. H. Dimmelmeier et al., "Gravitational waves from relativistic rotational core collapse", http://xxx.lanl.gov/abs/astro-ph/0103088, Astrophys.J. 560 L163-L166 (2001)

21. T. Pradier et al., "About the Detection of Gravitational Wave Bursts", http://xxx.lanl.gov/abs/gr-qc/0001062, Int.J.Mod.Phys. D9 309-314 (2000)

22. N. Arnaud et al., "Detection of gravitational wave bursts by interferometric detectors", http://xxx.lanl.gov/abs/gr-qc/9812015, Phys. Rev. D59 (1999) 082002 
23. N. Arnaud et al., "Triggers for the Detection of Gravitational Wave Bursts", http://xxx.lanl.gov/abs/grqc/9903035, Proceedings of the XXXIVth Rencontres de Moriond on "Gravitational Waves and Experimental Gravity" (1999)

24. T. Pradier et al., "An efficient filter for detecting gravitational wave bursts in interferometric detectors", http://xxx.lanl.gov/abs/gr-qc/0010037, Phys. Rev. D63 (2001)

25. N. Arnaud et al., "Detection in coincidence of gravitational wave bursts with a network of interferometric detectors (I): Geometric acceptance and timing", http://xxx.lanl.gov/abs/gr-qc/0107081, Phys. Rev. D65 (2002)

26. N. Arnaud et al., "Gravity Wave and Neutrino Bursts from Stellar Collapse: A Sensitive Test of Neutrino Masses", http://xxx.lanl.gov/abs/hep-ph/0109027, Phys. Rev. D65 (2002)

27. K. Scholberg, "SNEWS: The Supernova Early Warning System", preprint http://xxx.lanl.gov/abs/astro$\mathrm{ph} / 9911359$; the SNEWS web page, http://hep.bu.edu/ $\sim$ snnet

28. L. Robinson et al., Sky and Telescope, 98-2, 30, 8, August 1999. J. Bahcall et al., HST proposal \# 8404 cycles 8 and 9.

29. J. Beacom, and P. Vogel, "Can a supernova be located by its neutrinos?", http://xxx.lanl.gov/abs/astroph/9811350, Phys. Rev. D, 60, 033007, (1999)

30. D. Fargion, "Time Delay Between Gravitational Waves and Neutrino Burst From a Supernova Explosion: a Test for the Neutrino Mass", http://xxx.lanl.gov/abs/hep-ph/0110061, Lett.Nuovo Cim. 31 499-500 (1981)

31. H. Cuesta, "Gravitational-Wave Bursts Induced by Neutrino Oscillations: The Origin of Asymmetry in Supernovae Explosions", http://xxx.lanl.gov/abs/astro-ph/9904200

32. T. Piran, "Gamma-Ray Bursts - a Primer For Relativists", http://xxx.lanl.gov/abs/gr-qc/0205045, Review talk given at GR16

33. T. Piran, "Gravitational Radiation from Gamma-Ray Bursts", http://xxx.lanl.gov/abs/astro-ph/0102315

34. J. Buckley et al., "Gamma-Ray Summary Report”, http://xxx.lanl.gov/abs/astro-ph/0201160

35. M. van Putten, "Gravitational radiation from long gamma-ray bursts", http://xxx.lanl.gov/abs/gr-qc/0102043

36. M. van Putten and A. Levinson, "Calorimetry of gamma-ray bursts: echos in gravitational waves", http://xxx.lanl.gov/abs/astro-ph/0105429, Astrophys.J. 555 L41-L44 (2001)

37. M. van Putten, "Gravitational radiation from a torus around a black hole", http://xxx.lanl.gov/abs/astro$\mathrm{ph} / 0107007$

38. M. van Putten, "Gamma-ray bursts and gravitational radiation from black hole-torus systems", http://xxx.lanl.gov/abs/astro-ph/0109429

39. M. van Putten, "Superradiance in a Torus Magnetosphere Around a Black Hole", Science, Volume 284, Number 5411, Issue of 2 Apr 1999, pp. 115-118 (1999)

40. M. van Putten, "LIGO/VIRGO searches for gravitational radiation in hypernovae", http://xxx.lanl.gov/pdf/astro-ph/0207242

41. M. Davies et al., "Gamma-ray bursts, supernova kicks, and gravitational radiation", http://xxx.lanl.gov/abs/astro-ph/0204358

42. L. Fryer et al., "Pair Instability Supernovae, Gravity Waves, and Gamma-Ray Transients", http://xxx.lanl.gov/abs/astro-ph/0007176, Astrophys.J. 550 372-382 (2001)

43. S. Finn et al., "Detecting an association between Gamma Ray and Gravitational Wave Bursts", http://xxx.lanl.gov/abs/gr-qc/9903101, Phys. Rev. D60 (1999)

44. G. Modestino and A. Moleti, "On the cross correlation between Gravitational Wave Detectors for detecting association with Gamma Ray Bursts", http://xxx.lanl.gov/abs/astro-ph/0110620, Phys. Rev. D65 (2002)

45. P. Astone et al., "Search for correlation between GRB's detected by BeppoSAX and gravitational wave detectors EXPLORER and NAUTILUS", http://xxx.lanl.gov/abs/astro-ph/0206431

46. P. Tricarico et al., "Correlation between Gamma-Ray bursts and Gravitational Waves", http://xxx.lanl.gov/abs/gr-qc/0101022, Phys. Rev. D63 (2001) 